\title{
Overweight and Lonely? A Representative Study on Loneliness in Obese People and Its Determinants
}

\author{
Franziska U. Jung ${ }^{a-c} \quad$ Claudia Luck-Sikorski ${ }^{a, b}$ \\ antegrated Research and Treatment Center (IFB) Adiposity Diseases, University Hospital \\ Leipzig, Leipzig, Germany; ${ }^{b}$ SRH University of Applied Health Sciences, Gera, Germany; \\ 'Institute of Social Medicine, Occupational Health and Public Health, University of Leipzig, \\ Leipzig, Germany
}

\section{Keywords}

Obesity $\cdot$ Loneliness $\cdot$ Depression · Weight stigma

\begin{abstract}
Obesity is associated with physical and medical restrictions and comorbidities, but it also entails psychosocial effects such as social isolation and feelings of rejection. The aim of this study was to investigate the link between loneliness and weight stigma in a large sample of obese individuals. Results were derived from a large representative sample $(n=1,000)$. The survey included the 3-item version of the UCLA loneliness scale, the Patient Health Questionnaire (PHQ-9) as well as the Weight Bias Internalization scale (WBIS). The mean UCLA score was $1.943(S D=0.771)$. Respondents with higher levels of depression $(B=0.176)$, higher internalized weight bias $(B=0.435)$, and the experience of discrimination $(B=0.286)$ reported higher levels of loneliness. Future studies should investigate the mediation pathways between obesity, loneliness, and its determinants to provide a framework for successful interventions as part of obesity management programs.

(C) 2019 The Author(s)

Published by S. Karger AG, Basel
\end{abstract}

\section{Introduction}

Overweight and obesity are viewed as a major challenge for public health. According to estimations, around $38 \%$ of the adult population worldwide will be overweight and another $20 \%$ will be obese by 2030 , and the rate will be as high as $45 \%$ in the USA by $2035[1,2]$. Obese people face numerous somatic comorbidities and a shortened life expectancy [3], but they are also prone for social exclusion because of societies' body norms. Weight bias includes pervasive 
negative attributes and prejudice related to an individual's overweight, and they include stereotypes such as being lazy or incompetent [4]. Negative attitudes towards obese people have been documented worldwide [5], they are correlated with further weight gain and, therefore, exacerbated comorbidities. When those affected by stigmatization from their social environment agree with the negative attitudes and blame themselves for being overweight, an internalization of the stigmatizing attitudes has occurred [4]. A substantial number of obese people internalize these attitudes [6]. Discrimination and internalized stigma have been linked to depression, anxiety, and other psychological correlates [7, 8]. Often, experiencing discrimination and stigmatization, as well as depressive symptoms, may lead to social isolation. Previous studies $[9,10]$ indicated that people with depression are often affected by social distancing when it comes to occupational and interpersonal situations. Another study [11] showed that this also applies to obese people. The question remains whether people who face distancing also experience loneliness.

Several studies have provided evidence that loneliness can be found especially in elderly people. However, recent studies claim that it is not solely a question of age [12], but it can affect other vulnerable groups as well. Loneliness has been shown to be correlated with increased morbidity and mortality [13] as well as with many diseases such as a greater cardiovascular risk (reported, e.g., in Hawkley et al. [14]), decreased physical activity [15], self-reported poor sleep quality and daytime dysfunction (i.e., low energy, fatigue) [16]. All of these are factors that also play a role in the development and chronification of obesity. Few studies have investigated the role of loneliness in obesity. A very recent study [17] found a positive causal effect of BMI on loneliness but not for loneliness on BMI, not including weight bias as a variable. In a study on the impact of weight stigma and loneliness on suicidal behavior in bariatric patients, Chen et al. [18] showed that greater weight bias was associated with greater loneliness in both men and women. Another very recent study [19] examined the relationship between BMI and social withdrawal syndrome and loneliness. In that study, obese participants demonstrated lower emotional trust in close others, lower disclosure to close others, and greater loneliness compared to normal-weight or overweight participants. Whether this may be the result of experiencing discrimination due to weight has not been thematized in that study.

The aim of this study is, therefore, to investigate to what extent individuals with obesity of varying degree suffer from loneliness and, additionally, to determine the role of depression and weight stigma in this context. It is hypothesized that a substantial amount of obese people experience loneliness and that experiences of weight bias and depressive symptoms are positively correlated to feeling lonely.

\section{Method}

This study was approved by the local Ethics Committee of the University of Leipzig (vote number: 208-14-14042014). It was conducted with the help of a German research institute (Forsa). The data under investigation were derived from a large representative telephone survey.

\section{Participant Recruitment}

The data collection was conducted by a research institute (Forsa) that is specialized in surveys that focus on health-care topics. Due to their pool of participants that take part in Forsa's daily omnibus surveys, Forsa is also able to offer telephone interviews with partial groups that agree to take part on a regular basis. Representativeness was ensured using the Kish selection grid: a predefined randomization list is used here to determine the household member to be invited to the survey. By taking advantage of this panel that has already been recruited, the research institute was then able to directly contact participants with a BMI over $30 \mathrm{~kg} / \mathrm{m}^{2}$ only. About half did not want to be recontacted $(n=1,546)$, and others were not 
Jung and Luck-Sikorski: Obesity and Loneliness

reached, refused participation upon recontacting, or dropped out for other reasons. Overall, 1,000 telephone interviews were conducted by the institute's qualified interviewers (response rate: $45.6 \%$ ). Weighing variables were developed to ensure representativeness. The institute was responsible for documentation of consent of each participant during the interview. Participants did not receive any compensation.

\section{Instruments}

Forsa used questionnaires that were administered via telephone. They are self-report measures.

Loneliness

The main variable of interest, loneliness, was measured with the short 3-item version of the UCLA loneliness scale, using a 5-point scale [20]. For each item, the score will be added up and divided by the number of items. Higher scores reflect greater loneliness. The 3 -item version has shown satisfactory reliability and applicability in telephone surveys. In the present survey, the scale's Cronbach's $\alpha$ was 0.682 and, therefore, comparable to the original validation study $(0.72,[21])$ Additionally, the UCLA 3-item version is also used in the largest panel study of the German population, leaving the possibility for comparison with the general public [12]. In this study, the mean UCLA score was $M=0.99$. Other studies in older participants found higher mean scores $(M=4.06[22])$.

Depression

As covariate, depression was assessed with the Patient Health Questionnaire (PHQ-9, [23]), an instrument that can be used for the dimensional and categorical diagnosis of depression and depressive symptoms. Cronbach's $\alpha$ was 0.769 in the present sample.

Subjective Health

The general health status was assessed with an analogue scale from 0 to 100 (EQ-VAS, [24]). The participants were asked to choose one point on this scale, which represented their subjective health on that day. Higher scores indicate better self-rated health.

Weight Bias Internalization and Discrimination

Moreover, weight bias internalization was assessed by administering the German version of the Weight Bias Internalization scale (WBIS) [25]. Cronbach's $\alpha$ was 0.833 for this scale. Additionally, the Lifetime Discrimination Scale from the National Survey of Midlife Development in the US (MIDUS) on self-perceived discriminatory experiences was included to determine the frequency of experiences of discrimination in everyday life [26]. In addition, three more items were added to the original scale to specifically focus on weight-related discrimination. These items were generated through extensive qualitative research beforehand (unpublished results) and can be obtained from the authors. The items exemplify typical situations of weight discrimination (for instance "Have you been treated unfair in your work environment because of your weight?") and dichotomizing experiences of discrimination throughout the life span (yes/no).

Sociodemographic Variables

Sociodemographic variables were assessed with a standardized procedure provided by the research institute, including age, gender, and education. As loneliness has been reported to occur more often in people living alone [21], it was also assessed whether our participants live alone or with someone (i.e., a partner, family member, or friend). 
Jung and Luck-Sikorski: Obesity and Loneliness

\section{Statistical Analysis}

All analyses were conducted using STATA 13.1. Whenever possible, variables were used as continuous predictors (age, depressive symptoms, health status, and weight bias internalization). Where feasible (e.g., for discrimination experiences or living together with someone), a dichotomized variable was constructed to reflect the presence or absence of the relevant construct. BMI was further categorized into three groups according to WHO recommendations [27]: obesity class I $\left(30.0-34.9 \mathrm{~kg} / \mathrm{m}^{2}\right)$, obesity class II $\left(35.0-39.9 \mathrm{~kg} / \mathrm{m}^{2}\right)$, and obesity class III $\left(\geq 40.0 \mathrm{~kg} / \mathrm{m}^{2}\right)$. In the descriptive analysis, the $\chi^{2}$ test or one-way ANOVA was conducted to assess differences across obesity classes. The results from a representative study in Germany were used to assess differences in levels of loneliness between the study that included participants from all weight groups [12] and the present study. The mean of the UCLA loneliness scale in this study was $M=0.99$ [12]. A one-sample mean comparison test ( $t$ test) was used. The relevant $p$ value was set at $<0.05$.

Loneliness was used as the dependent continuous variable in linear regression models. All independent variables were introduced in univariate models as well as together in a full model. Standardized coefficients are reported. Predictor effect sizes were assessed through proportions of variance explained (STATA command estat esize). Interactions of variables were investigated and only included when significant. In particular, the interactions of weight bias internalization and depressive symptoms or discrimination experiences, as well as internalization and living with someone, were tested. These were derived from the literature in the field. The research institute (Forsa) also provided a variable to weigh data according to the German public. All analyses were run with weighted and non-weighted data, and the results are reported.

\section{Results}

Table 1 summarizes all variables of interests and reports differences across the three obesity classes in non-weighted data as no differences were found when applying the weighing variable. In total, $55.2 \%$ of the respondents were male, with a mean age of 56.4 years, and were more often living with someone than alone (62\%). Almost $40 \%$ reported weight-based discrimination (39.8\%). The mean loneliness score was significantly higher in our sample $(M=1.9)$ compared to a recent analysis in the German public $(M=0.99, p<$ $0.001,[12])$. While no differences were observed regarding loneliness - with the comparison of participants with obesity class I and II being borderline significant at $p=0.054-$, participants differed in other relevant variables. Participants with higher BMI classes reported higher levels of depression, higher internalization of weight bias, and suffered discrimination experiences more often. They also less likely lived with someone. Furthermore, the self-reported health status was significantly worse among those participants with higher BMI categories.

Table 2 reports the results from univariate and multivariate regression models. The full model explained $27.1 \%$ of the variance. Significant associations were found for four variables: participants living with someone reported lower levels of loneliness, while respondents with higher levels of depression, higher internalized weight bias, and the experience of discrimination experiences reported higher levels of loneliness. No interaction terms were significant. Effect sizes for the significant variables varied: depressive symptoms explained the largest proportion of variance (0.091), followed by living together with someone (0.027), and weight bias internalization (0.052). The presence of discrimination experiences only explained $0.03 \%$ of the variance, and the $95 \%$ confidence interval overlapped for this variable. 
Table 1. Descriptive statistics for all variables for the total sample and by BMI group

\begin{tabular}{|c|c|c|c|c|c|}
\hline \multirow[t]{2}{*}{ Variable } & \multirow{2}{*}{$\begin{array}{l}\text { Total sample } \\
(n=1,000)\end{array}$} & \multicolumn{3}{|l|}{ BMI } & \multirow[t]{2}{*}{$p$ value } \\
\hline & & $\begin{array}{l}30-34.9 \mathrm{~kg} / \mathrm{m}^{2} \\
(\mathrm{I} ; n=671)\end{array}$ & $\begin{array}{l}35-39.9 \mathrm{~kg} / \mathrm{m}^{2} \\
(\mathrm{II} ; n=245)\end{array}$ & $\begin{array}{l}\geq 40 \mathrm{~kg} / \mathrm{m}^{2} \\
\text { (III; } n=84 \text { ) }\end{array}$ & \\
\hline Gender & & & & & 0.099 \\
\hline Women & 44.8 & 42.9 & 46.5 & 54.7 & \\
\hline Men & 55.2 & 57.8 & 53.5 & 45.2 & \\
\hline Living situation & & & & & 0.025 \\
\hline Alone & 38.0 & 35.6 & 40.4 & 50.0 & \\
\hline With someone & 62.0 & 64.4 & 59.6 & 50.0 & \\
\hline \multicolumn{6}{|c|}{ Discrimination experience (MIDUS) } \\
\hline Yes & 39.8 & 32.3 & 47.8 & 76.2 & $<0.001$ \\
\hline No & 60.2 & 67.7 & 52.2 & 23.8 & \\
\hline Age & $56.4 \pm 14.9$ & $57.6 \pm 15.0$ & $54.7 \pm 14.5$ & $52.4 \pm 14.1$ & $\begin{array}{l}\text { I vs. II: } 0.033 \\
\text { I vs. III: } 0.010\end{array}$ \\
\hline $\begin{array}{l}\text { Depressive symptoms } \\
\text { (PHQ) }\end{array}$ & $14.3 \pm 4.30$ & $14.0 \pm 4.0$ & $14.9 \pm 4.5$ & $15.1 \pm 4.9$ & I vs. II: 0.020 \\
\hline $\begin{array}{l}\text { Weight Bias Internal- } \\
\text { ization scale (WBIS) }\end{array}$ & $27.5 \pm 11.9$ & $26.3 \pm 11.5$ & $28.3 \pm 11.9$ & $34.1 \pm 13.1$ & $\begin{array}{l}\text { I vs. III: }<0.001 \\
\text { II vs. III: } 0.001\end{array}$ \\
\hline Health status (VAS) & $63.7 \pm 22.6$ & $66.0 \pm 21.4$ & $20.0 \pm 23.2$ & $55.8 \pm 26.8$ & $\begin{array}{l}\text { I vs. II: } 0.002 \\
\text { I vs. III: } 0.001\end{array}$ \\
\hline Loneliness (range: $1-5$ ) & $1.9 \pm 0.8$ & $1.9 \pm 0.7$ & $2.0 \pm 0.8$ & $2.0 \pm 0.8$ & ns \\
\hline
\end{tabular}

Values are given as percentage or mean \pm SD. $p$ values are calculated using $\chi^{2}$ test (categorial variables) or one-way ANOVA (dimensional variables). ns, not significant.

\section{Discussion}

According to the current study, a higher BMI can be associated with higher levels of depression, higher internalization of weight bias, and a higher rate of discrimination experiences. Furthermore, the self-reported health status was significantly worse among those participants with higher BMI categories. This could account for the generally high level of loneliness observed in this sample, as obesity has been linked to worse self-reported health only in the presence of high levels of loneliness [28]. When comparing weight categories, obese individuals are in general lonelier in the current sample, regardless of their class of obesity. This has been shown in previous studies as well [29]. Secondly, with regard to loneliness, participants with higher levels of depression, higher internalized weight bias, and the experience of discrimination reported higher levels of loneliness.

Obesity and loneliness seem to be connected by acting as a negative synergy on both physical and emotional well-being. A few studies argue that loneliness might increase obesity, for instance by negatively affecting quality of sleep. In this context, sleep may be disrupted due to night-eating symptoms that also occur in the presence of obesity [30] and could, therefore, predict further weight gain. Alterations on the HPA-axis (i.e., related to psychosocial stress) have been shown to be associated with loneliness [31] as well as weight stigmatization and discrimination [32] and may promote weight gain by increasing eating behavior and abdominal adiposity [33]. The mediation pathways between obesity, stigma, and loneliness are still not known and should, therefore, be investigated in future studies. 
Table 2. Regression coefficients in univariate and multivariate regression models (dependent variable: loneliness)

\begin{tabular}{|c|c|c|c|c|}
\hline \multirow[t]{2}{*}{ Variable } & \multicolumn{2}{|c|}{ Univariate model } & \multicolumn{2}{|l|}{ Full model } \\
\hline & B & SE & $\mathrm{B}$ & SE \\
\hline \multicolumn{5}{|l|}{ Gender } \\
\hline Men & - & & - & \\
\hline Women & 0.749 & 0.049 & -0.205 & 0.132 \\
\hline \multicolumn{5}{|l|}{ Living situation } \\
\hline Alone & - & & - & \\
\hline With someone & $-0.337^{* * *}$ & 0.049 & $-0.706^{* * *}$ & 0.135 \\
\hline \multicolumn{5}{|l|}{ Discrimination experience } \\
\hline No & - & & - & \\
\hline Yes & $0.335^{* * *}$ & 0.049 & $0.286^{*}$ & 0.143 \\
\hline \multicolumn{5}{|l|}{ BMI category } \\
\hline $30-34.9 \mathrm{~kg} / \mathrm{m}^{2}$ & - & & - & \\
\hline $35-39.9 \mathrm{~kg} / \mathrm{m}^{2}$ & $0.139 *$ & 0.058 & 0.128 & 0.155 \\
\hline$\geq 40 \mathrm{~kg} / \mathrm{m}^{2}$ & 0.137 & 0.089 & -0.356 & 0.246 \\
\hline Age & -0.002 & 0.001 & -0.003 & 0.005 \\
\hline Depressive symptoms (PHQ) & $0.079 * * *$ & 0.005 & $0.176^{* * *}$ & 0.018 \\
\hline Weight Bias Internalization scale (WBIS) & $0.024^{* * *}$ & 0.002 & $0.435^{* * *}$ & 0.006 \\
\hline Health status (VAS) & $-0.007^{* * *}$ & 0.001 & -0.003 & 0.003 \\
\hline
\end{tabular}

\section{Strengths and Limitations}

This study is a cross-sectional observational study and cannot explain causality. Although a standardized measure was used for loneliness, an in-depth assessment of the constructs could not be done. BMI was based on self-reports only. Also, the comparison with a large representative study across all weight categories is limited since age could not be harmonized between the two studies. However, this is one of the largest samples of obese people living in Germany, which is representative of the German public. It is therefore a valuable epidemiological addition to the literature.

\section{Conclusions}

Overall, the results of the current study suggest that loneliness may occur more often in obese individuals and that it is related to depressive symptoms, greater self-stigma, and weight discrimination. Social isolation or loneliness is known to be a risk factor for precarious health and greater mortality. Loneliness is also linked to obesity; however, cause and effect in this relationship still remain unclear. It may be seen as a complication or a trigger for obesity. Therefore, loneliness should be acknowledged as a public health challenge that needs to be addressed in terms of multidisciplinary weight management. Avoidance of medical care (i.e., due to social withdrawal or a lack of trust in others) could increase the burden and lower overall quality of life. On the other hand, it may lower the incidence of help-seeking behavior in terms of weight management. If people understand the complexity of this disease, stigmatization and discrimi- 
nation may be eliminated and, thereby, reduce social distancing. On the other hand, if obese individuals feel understood and accepted, they may not fear social situations as a threat [34,35]. It could be one way to support those you are vulnerable to weight bias by increasing trust and helping them to build up social networks, because social relationships are important not only for physical health but also for emotional and psychological well-being.

\section{Statement of Ethics}

This study was approved by the local Ethics Committee of the University of Leipzig (vote number: 208-14-14042014).

\section{Disclosure Statement}

Both authors declare to have no conflicts of interest.

\section{Funding Sources}

This work was supported by the Federal Ministry of Education and Research (BMBF), Germany, FKZ: 01E01501. The authors acknowledge support from the German Research Foundation (DFG) and Universität Leipzig within the program of Open Access Publishing.

\section{Author Contributions}

F.U.J. wrote the first draft of the manuscript as well as made the (internal) revision of the manuscript in collaboration with C.L.-S., who was also responsible for data collection and data analysis.

\section{References}

1 Kelly T, Yang W, Chen CS, Reynolds K, He J. Global burden of obesity in 2005 and projections to 2030. Int J Obes. 2008 Sep;32(9):1431-7.

2 Revels S, Kumar SA, Ben-Assuli O. Predicting obesity rate and obesity-related healthcare costs using data analytics. Health Policy Technol. 2017;6(2):198-207.

3 Abdelaal M, le Roux CW, Docherty NG. Morbidity and mortality associated with obesity. Ann Transl Med. 2017 Apr;5(7):161.

4 Puhl RM, Heuer CA. The stigma of obesity: a review and update. Obesity (Silver Spring). 2009 May;17(5):941-64.

5 Macmillan M. The future of television and medicine. Proc R Soc Med. 1969 Apr;62(4):401-2.

6 Puhl RM, Himmelstein MS, Quinn DM. Internalizing Weight Stigma: Prevalence and Sociodemographic Considerations in US Adults. Obesity (Silver Spring). 2018 Jan;26(1):167-75.

7 Papadopoulos S, Brennan L. Correlates of weight stigma in adults with overweight and obesity: A systematic literature review. Obesity (Silver Spring). 2015 Sep;23(9):1743-60.

8 Sikorski C, Luppa M, Luck T, Riedel-Heller SG. Weight stigma "gets under the skin"-evidence for an adapted psychological mediation framework: a systematic review. Obesity (Silver Spring). 2015 Feb;23(2):266-76.

9 Angermeyer MC, Matschinger H. Social distance towards the mentally ill: results of representative surveys in the Federal Republic of Germany. Psychol Med. 1997 Jan;27(1):131-41.

10 Angermeyer MC, Matschinger H, Schomerus G. Public attitudes towards people with depression in times of uncertainty: results from three population surveys in Germany. Soc Psychiatry Psychiatr Epidemiol. 2013 Sep; 48(9):1513-8. 
Jung and Luck-Sikorski: Obesity and Loneliness

11 Sikorski C, Luppa M, Angermeyer MC, Schomerus G, Link B, Riedel-Heller SG. The association of BMI and social distance towards obese individuals is mediated by sympathy and understanding. Soc Sci Med. 2015 Mar; 128: $25-30$.

12 Luhmann M, Hawkley LC. Age differences in loneliness from late adolescence to oldest old age. Dev Psychol. 2016 Jun;52(6):943-59.

13 Rico-Uribe LA, Caballero FF, Martín-María N, Cabello M, Ayuso-Mateos JL, Miret M. Association of loneliness with all-cause mortality: A meta-analysis. PLoS One. 2018 Jan;13(1):e0190033.

14 Hawkley LC, Thisted RA, Masi CM, Cacioppo JT. Loneliness predicts increased blood pressure: 5-year crosslagged analyses in middle-aged and older adults. Psychol Aging. 2010 Mar;25(1):132-41.

15 Hawkley LC, Thisted RA, Cacioppo JT. Loneliness predicts reduced physical activity: cross-sectional \& longitudinal analyses. Health Psychol. 2009 May;28(3):354-63.

16 Hawkley LC, Preacher KJ, Cacioppo JT. Loneliness impairs daytime functioning but not sleep duration. Health Psychol. 2010 Mar;29(2):124-9.

17 Day FR, Ong KK, Perry JR. Elucidating the genetic basis of social interaction and isolation. Nat Commun. 2018 Jul;9(1):2457.

18 Chen EY, Fettich KC, McCloskey MS. Correlates of suicidal ideation and/or behavior in bariatric-surgeryseeking individuals with severe obesity. Crisis. 2012 Jan;33(3):137-43.

19 Rotenberg KJ, Bharathi C, Davies H, Finch T. Obesity and the Social Withdrawal Syndrome. Eat Behav. 2017 Aug;26:167-70.

20 Hughes ME, Waite LJ, Hawkley LC, Cacioppo JT. A Short Scale for Measuring Loneliness in Large Surveys: Results From Two Population-Based Studies. Res Aging. 2004;26(6):655-72.

21 Huschtscha L, Bodmer W. The isolation and description of LIF-10: a human tumor cell line derived from a colorectal adenocarcinoma. In Vitro Cell Dev Biol. 1990 Aug;26(8):743-5.

22 Steptoe A, Shankar A, Demakakos P, Wardle J. Social isolation, loneliness, and all-cause mortality in older men and women. Proc Natl Acad Sci USA. 2013 Apr;110(15):5797-801.

23 Kroenke K, Spitzer RL, Williams JB. The PHQ-9: validity of a brief depression severity measure. J Gen Intern Med. 2001 Sep;16(9):606-13.

24 Graf J, Claes C, Greiner W, Uber A. Die deutsche Version des EuroQol-Fragebogens. J Public Health (Bangkok). 1998;6(1):3-20.

25 Hilbert A, Baldofski S, Zenger M, Löwe B, Kersting A, Braehler E. Weight bias internalization scale: psychometric properties and population norms. PLoS One. 2014 Jan;9(1):e86303.

26 Williams DR, Yan Yu, Jackson JS, Anderson NB. Racial Differences in Physical and Mental Health: Socioeconomic Status, Stress and Discrimination. J Health Psychol. 1997 Jul;2(3):335-51.

27 World Health Organization. Obesity: preventing and managing the global epidemic: report of a WHO Consultation. WHO Technical Report Series 894. Geneva: WHO; 2000.

28 Tigani X, Artemiadis AK, Alexopoulos EC, Chrousos GP, Darviri C. Self-rated health in centenarians: a nationwide cross-sectional Greek study. Arch Gerontol Geriatr. 2012 May-Jun;54(3):e342-8.

29 Lauder W, Mummery K, Jones M, Caperchione C. A comparison of health behaviours in lonely and non-lonely populations. Psychol Health Med. 2006 May;11(2):233-45.

30 Morse SA, Ciechanowski PS, Katon WJ, Hirsch IB. Isn't this just bedtime snacking? The potential adverse effects of night-eating symptoms on treatment adherence and outcomes in patients with diabetes. Diabetes Care. 2006 Aug;29(8):1800-4.

31 Brown EG, Gallagher S, Creaven AM. Loneliness and acute stress reactivity: A systematic review of psychophysiological studies. Psychophysiology. 2018 May;55(5):e13031.

32 Himmelstein MS, Incollingo Belsky AC, Tomiyama AJ. The weight of stigma: cortisol reactivity to manipulated weight stigma. Obesity (Silver Spring). 2015 Feb;23(2):368-74.

33 Scott KA, Melhorn SJ, Sakai RR. Effects of Chronic Social Stress on Obesity. Curr Obes Rep. 2012 Mar;1(1): $16-25$.

34 Tomiyama AJ. Weight stigma is stressful. A review of evidence for the Cyclic Obesity/Weight-Based Stigma model. Appetite. 2014 Nov;82:8-15.

35 Major B, O’Brien LT. The social psychology of stigma. Annu Rev Psychol. 2005;56(1):393-421. 